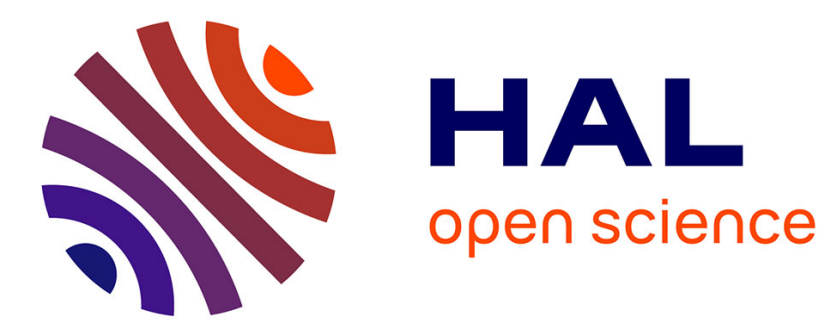

\title{
Reflection on a rough surface from an acoustic point source
}

Maurice A. Biot

\section{To cite this version:}

Maurice A. Biot. Reflection on a rough surface from an acoustic point source. Journal of the Acoustical Society of America, 1957, 29 (11), pp.1193-1200. 10.1121/1.1908741 . hal-01368682

\section{HAL Id: hal-01368682 \\ https://hal.science/hal-01368682}

Submitted on 21 Sep 2016

HAL is a multi-disciplinary open access archive for the deposit and dissemination of scientific research documents, whether they are published or not. The documents may come from teaching and research institutions in France or abroad, or from public or private research centers.
L'archive ouverte pluridisciplinaire HAL, est destinée au dépôt et à la diffusion de documents scientifiques de niveau recherche, publiés ou non, émanant des établissements d'enseignement et de recherche français ou étrangers, des laboratoires publics ou privés. 


\title{
Reflection on a Rough Surface from an Acoustic Point Source
}

\author{
M. A. Bioт* \\ Shell Development Company, New York, New York
}

(Received April 29, 1957)

\begin{abstract}
A simple solution is developed for the reflected waves on a rough surface from a simple harmonic point source. It is assumed that the roughness is represented by a distribution of hemispherical bosses whose size and mutual distance are small relative to the wavelength. It is shown that under these conditions the effect of the roughness is equivalent to a boundary condition for the wave equation. This boundary condition embodies the surface polarization and the mutual interaction of the bosses. If the generating source lies above the reflecting surface the reflected wave is found to be equivalent to that originating from concentrated and distributed image sources on a line situated below the specular image with a magnitude decreasing exponentially with depth. The case of vanishingly small roughness is discussed along with the field intensity at large distance and grazing incidence. The effect of fluid viscosity is also evaluated.
\end{abstract}

\section{INTRODUCTION}

$W^{3}$ $\mathrm{E}$ are concerned here with a simple solution to the problem of reflection of acoustic waves from a rough surface under certain assumptions. When a simple harmonic wave strikes a rough surface on which the roughness is represented by distributed protuberances, the influence of this roughness may be separated into a coherent reflected wave and an incoherent part. The latter represents the scatter proper. If, however, the size of the protuberances and their mutual distance become small relative to the protuberances and their mutual distance become small relative to the wavelength the coherent reflection plays the dominant role. It is this particular aspect of the phenomenon which we are dealing with in the present treatment of the problem. Another equivalent viewpoint is to look upon this phenomenon as due to a two-dimensional surface polarization induced in the protuberances by the incident wave and simultaneously by the interaction of waves emitted by the protuberances themselves. This interaction plays an important role and modifies essentially the character of the reflection even for very small size roughness.

The particular acoustic problem considered here is that of the reflection of simple harmonic waves emanating from a point source situated at a given distance $h$ above the reflecting surface. The procedure followed is entirely analogous to that established by this writer in some earlier work for the reflection on a rough surface of plane electromagnetic and acoustic ${ }^{1}$ waves, and of an electromagnetic dipole source. ${ }^{2}$ The solution for the reflected wave is in closed form. The roughness is represented by a distribution of hemispherical bosses on a plane. One advantage of this representation is its

\footnotetext{
* Consultant.

1 M. A. Biot, "Reflection of an electromagnetic plane wave from a surface with small roughness," Cornell Aeronautical Laboratory Report (March, 1955) [to be published in part under the title "Some new aspects of the reflection of electromagnetic waves on a rough surface," J. Appl. Phys. (to be published)].

${ }_{2} \mathrm{M}$. A. Biot, "A closed form solution for the reflection of electromagnetic dipole radiation on a rough surface," Cornell Aeronautical Laboratory Report, March, 1955 (to be published).
}

inclusion in the theory of the effect of vertical slope of the protuberances. The essential feature of our procedure which was introduced in the earlier work $^{1,2}$ lies in the substitution of the integral equation representing the interaction of the bosses by a boundary condition for the wave equation. This boundary condition is simply a linear relation between the first and second normal derivatives of the velocity potential. This opens the way to a very flexible and simple treatment of reflection and diffraction problems from and around rough obstacles. Similarly, the case of nonuniform roughness may be introduced by varying the boundary condition from point to point.

Use of spherical and cylindrical protuberances to represent surface roughness has been made by Twersky ${ }^{3}$ in evaluations of the scattering cross section for plane waves. The emphasis is on energy evaluation of the scattered field. In a more recent paper ${ }^{4}$ he also derives the existence of a phase reversal at grazing incidence in accordance with our previous results for plane waves ${ }^{1}$ and the present formulas for the far field in the case of a point source. In Sec. 2 we derive the boundary condition which is equivalent to the effect of the roughness. From this boundary condition and the Sommerfeld representation of an acoustic point source an expression is derived in Sec. 3 for the reflected field. In Sec. 4 it is shown that if the source lies above a horizontal plane of reflection the reflected field is represented by the radiation of a concentrated image and exponentially distributed images lying on a vertical line below the specular image of the source.

Several special cases are discussed in Sec. 5. The case of vanishingly small roughness is considered and it is shown that in that case the reflected wave results from a specular image and a distribution of images of very small intensity lying on the vertical line below the specular image over a wide range of depth. This shows that for a pulse signal the small roughness

V. Twersky, J. Appl. Phys. 22, 852 (1951).

4 V. Twersky, J. Acoust. Soc. Am. 29, 209 (1957). 
induces a trail of small intensity echoes with large time lags. The reflected field at large distances, i.e., grazing incidence, is also discussed. It is shown that the incident plus reflected wave cancel each other in a region of grazing incidence. The extent of this zone of silence is evaluated in terms of the magnitude of the roughness. Finally, the effect of the fluid viscosity is evaluated by considering the thickness of the boundary layer near the rigid wall. It is found that in practice the viscosity will not generally have any appreciable influence.

The existence of a zone of silence at grazing incidence may be understood physically by the fact that the roughness induces a wave disturbance which is in phase opposition with the incident wave. The effect is cumulative at grazing incidence because the phase velocity of the incident wave along the reflecting surface is equal or very near to the velocity of propagation of the wave disturbance. For the effect to occur the disturbance due to the roughness must propagate in the same direction as the incident wave. This is exactly the case for the induced dipoles in the acoustic case. The physical significance of this effect was also discussed in reference 1 for the electromagnetic wave, where is was found that the existence of a zone of silence depends on the polarization. Another difference of the electromagnetic case with the acoustic case lies in the disappearance of the influence of the roughness near an angle of incidence of $45^{\circ}$. This is because, in the former case, two types of dipole disturbances are induced (electric and magnetic) and that their effects cancel each other at a particular angle of incidence.

When the distance between bosses becomes of the order of their diameter, a short range interaction appears which is nonradiative and may be taken into account by introducing a correction coefficient $\kappa$ close to unity. This point is developed in the appendix.

It will be remembered that the present treatment is for reflection from a rigid surface. A rough free surface, as for example that encountered by sound waves in the water at the ocean surface, should behave quite differently.

\section{REPLACEMENT OF THE ROUGH SURFACE BY A BOUNDARY CONDITION}

Let us first consider a rigid plane of reflection on which a single roughness element is located.

This element will be represented by a hemispherical boss of radius $a$. It is further assumed that the radius $a$ is small relative to the wavelength $\lambda$. This being the case we may consider the velocity caused by the incident and reflected wave on the plane surface in the absence of any roughness. We may assume this velocity field to be approximately uniform in a region of the order of magnitude of the radius $a$. Let us locate the boss at the origin, the $x, y$ plane being the reflecting plane, and the waves propagating in the half-space $z>0$. The boss produces a radiating disturbance represented by a dipole of intensity and orientation such that on the sphere of radius $a$ the normal component of the undisturbed field is canceled. With an undisturbed field of uniform velocity $U_{x}$ directed along $x$, the dipole axis lies in the same direction, and the velocity potential $\varphi^{\prime}$ generated by this dipole is

$$
\varphi^{\prime}=-\frac{1}{2} a^{3} U_{x} \frac{\partial}{\partial x}\left(\frac{e^{-i k R^{\prime}}}{R^{\prime}}\right)
$$

where $R^{\prime}=\left(x^{2}+y^{2}+z^{2}\right)^{\frac{1}{2}}, \omega=$ angular frequency, $k=\omega / c$, and $c=$ velocity of sound. Our assumption regarding the wavelength corresponds to $k a \ll 1$.

We may also write

with

$$
\varphi_{x}^{\prime}=\partial \phi_{x} / \partial x
$$

$$
\phi_{x}=-\frac{1}{2} a^{3} U_{x} \frac{e^{-i k R^{\prime}}}{R^{\prime}} .
$$

Similarly, when the undisturbed velocity field is directed along the $y$ direction and represented by the component $U_{y}$, the field radiated by the hemispherical boss is given by the velocity potential

$$
\varphi_{y}^{\prime}=\frac{\partial \phi_{y}}{\partial y},
$$

with

$$
\phi_{y}=-\frac{1}{2} a^{3} U_{y} \frac{e^{-i k R}}{R^{\prime}} .
$$

If the undisturbed field is in an arbitrary direction of velocity components $U_{x}$ and $U_{y}$ the velocity potential $\varphi^{\prime}$ of the disturbance is the superposition of (2.2) and (2.4) hence

$$
\varphi^{\prime}=\varphi_{x}{ }^{\prime}+\varphi_{y}{ }^{\prime}=\frac{\partial \phi_{x}}{\partial x}+\frac{\partial \phi_{y}}{\partial y} .
$$

We now consider the case when there is a continuous and uniform distribution of the roughness on the plane of reflection in the form of hemispherical bosses of radius $a$. The density of the distribution is represented by the number $N$ of these bosses per unit area.

At any point of the reflecting plane there is a velocity parallel with this plane and of components $U_{x}$ and $U_{y}$. Due to the presence of the bosses this field generates a disturbance which is the integrated effect of all the radiating dipoles on the surface of reflection. The velocity potential of the disturbances radiated by the continuous distribution of all the bosses is denoted by $\varphi$ and is obtained as follows. We multiply expressions (2.3) and (2.5) by the boss density $N$ and integrate 
over the $x, y$ plane, thus

$$
\begin{aligned}
& V_{x}=N \iint \phi_{x} d \xi d \eta=-\frac{1}{2} N a^{3} \iint U_{x} \frac{e^{-i k R}}{R} d \xi d \eta \\
& V_{y}=N \iint \phi_{y} d \xi d \eta=-\frac{1}{2} N a^{3} \iint U_{y} \frac{e^{-i k R}}{R} d \xi d \eta .
\end{aligned}
$$

The distance $R$ is now

$$
R=\left[(x-\xi)^{2}+(y-\eta)^{2}+z^{2}\right]^{2} .
$$

Actually we should write $(1 / \kappa) U_{x}$ and $(1 / \kappa) U_{y}$ instead of $U_{x}$ and $U_{y}$ in Eq. (2.7) where the coefficient $\kappa$ takes into account close range nonradiative interaction of the bosses. This amounts to putting $\sigma=2 \pi N a^{3} / \kappa$ (see following). The coefficient $\kappa$ is close to unity and is evaluated in the appendix. The velocity potential radiated by the bosses is then

$$
\varphi=\frac{\partial V_{x}}{\partial x}+\frac{\partial V_{y}}{\partial y} .
$$

The important step in this derivation is the transformation of the integral relations (2.7) into equivalent differential relations. The expressions $V_{x}$ and $V_{y}$ by their definitions (2.7) are represented by a distribution of sources in the $x, y$ plane. In a vanishingly small region near this plane the sources behave as sources of Newtonian potential. Therefore, applying Gauss's theorem the derivative of $V_{x}$ and $V_{y}$ normal to this plane is proportional to the local surface density of the sources. We find

$$
\begin{aligned}
& \left(\frac{\partial V_{x}}{\partial z}\right)_{z=0}=-\frac{1}{2} \sigma U_{x} \\
& \left(\frac{\partial V_{y}}{\partial z}\right)_{z=0}=-\frac{1}{2} \sigma U_{y},
\end{aligned}
$$

with

$$
\sigma=2 \pi N a^{3} .
$$

Combining these relations with Eq. (2.9) we derive the boundary condition at $z=0$

$$
\frac{\partial \varphi}{\partial z}=-\frac{1}{2} \sigma\left(\frac{\partial U_{x}}{\partial x}+\frac{\partial U_{y}}{\partial y}\right) .
$$

In expression (2.11) the velocity of components $U_{x}, U_{y}$ is unknown. It is the velocity resulting from,

1. The incident field of velocity potential $\varphi_{\mathrm{s}}$.

2. A specularly reflected field of velocity potential $\varphi_{i}$, which corresponds to reflection in the absence of roughness and is given by a specular image located at $z=-h$ and

3. The field of velocity potential $\varphi$ produced by the radiation of the bosses. It is part of the "undisturbed" field acting on a local boss as considered in Eqs. (2.3) and (2.5).

If $\phi$ is the total velocity potential resulting from the superposition of all three fields we write

$$
\phi=\varphi_{s}+\varphi_{i}+\varphi
$$

and it satisfies the wave equation

$$
\frac{\partial^{2} \phi}{\partial x^{2}}+\frac{\partial^{2} \phi}{\partial y^{2}}+\frac{\partial^{2} \phi}{\partial z^{2}}+k^{2} \phi=0 .
$$

This equation is also satisfied by the separate velocity potential $\varphi_{i}, \varphi_{s}$, and $\varphi$. The velocity components $U_{x}$ and $U_{y}$ are thus expressed by

$$
\begin{aligned}
& U_{x}=\partial \phi / \partial x \\
& U_{y}=\partial \phi / \partial y .
\end{aligned}
$$

Taking into account (2.13) we derive

$$
\frac{\partial U_{x}}{\partial x}+\frac{\partial U_{y}}{\partial y}=\frac{\partial^{2} \phi}{\partial x^{2}}+\frac{\partial^{2} \phi}{\partial y^{2}}=-\frac{\partial^{2} \phi}{\partial z^{2}}-k^{2} \phi .
$$

The boundary condition (2.11) at $z=0$ then becomes

$$
\frac{\partial \varphi}{\partial z}=\frac{1}{2} \sigma\left(\frac{\partial^{2}}{\partial z^{2}}+k^{2}\right)\left(\varphi_{i}+\varphi_{8}+\varphi\right) .
$$

Knowing the velocity potentials $\varphi_{s}$ and $\varphi_{i}$ for the incident and reflected fields in the absence of roughness the problem then reduces to finding a solution $\varphi$ of the wave Eq. (2.13) satisfying the boundary condition (2.16) and the Sommerfeld radiation condition. Since at the boundary

$$
\frac{\partial}{\partial z}\left(\varphi_{i}+\varphi_{s}\right)=0
$$

an equivalent way of writing the boundary condition is

$$
\frac{\partial \phi}{\partial z}=\frac{1}{2} \sigma\left(\frac{\partial^{2}}{\partial z^{2}}+k^{2}\right) \phi
$$

with the total velocity potential (2.12).

We must remember that the derivative $\partial / \partial z$ is the normal derivative to the surface taken positive in a direction moving away from the solid. From the way this boundary condition has been derived it is clear that it applies also to a curved surface provided the radius of curvature is large relative to the size of the roughness.

\section{CLOSED FORM SOLUTION FOR THE REFLECTED WAVE}

The source is assumed to be located on the $z$ axis at a distance $z=h$ from the reflecting plane $z=0$. The velocity potential of this source is

$$
\varphi_{\mathrm{s}}=D\left(e^{-i k R_{\mathrm{s}}} / R_{\mathrm{s}}\right)
$$


with

$$
\begin{aligned}
R_{8} & =\left[r^{2}+(z-h)^{2}\right]^{\frac{1}{3}}, \\
r^{2} & =x^{2}+y^{2},
\end{aligned}
$$

and where $D$ is a constant defining the magnitude of the source. Similarly, the specular image source located at $z=-h$ is represented by the velocity potential

with

$$
\varphi_{i}=D\left(e^{-i k R_{i} / R_{i}}\right)
$$

$$
R_{i}=\left[r^{2}+(z+h)^{2}\right]^{z} \text {. }
$$

Since the problem is axisymmetric, a cylindrical coordinate system is used.

In order to determine the unknown potential $\varphi$ we represent the field radiated by the source as a Sommerfeld integral. ${ }^{5,6}$

$$
\varphi_{s}=\frac{D e^{-i k R_{s}}}{R_{s}}=D \int_{0}^{\infty} \frac{1}{\mu} e^{-\mu|z-h|} J_{0}(l r) l d l,
$$

where

$$
\mu=\left(l^{2}-k^{2}\right)^{\frac{1}{2}}
$$

and $J_{0}$ is the Bessel function of the first kind of zero order. The sign of the radical is chosen so that $\mu$ is positive real or positive imaginary. Similarly the specular image is represented by

$$
\varphi_{i}=\frac{D e^{-i k R_{i}}}{R_{i}}=D \int_{0}^{\infty} \frac{1}{\mu} e^{-\mu|z+h|} J_{0}(l r) l d l .
$$

In the region

$$
-h<z<h
$$

the absolute value sign in the exponential factor may be dropped and we may write

$$
\varphi_{z}+\varphi_{i}=D \int_{0}^{\infty} \frac{1}{-} J_{0}(l r) e^{-\mu h}\left[e^{\mu z}+e^{-\mu z}\right] l d l .
$$

We now represent the unknown $\varphi$ by an integral of a similar type with a spectral function $F(l)$ to be determined. We write

$$
\varphi=\int_{0}^{\infty} \frac{1}{\mu} F(l) J_{0}(l r) e^{-\mu(z+h)} l d l .
$$

This expression satisfies the wave Eq. (2.13) and the Sommerfeld radiation condition since it represents radiation propagating in the positive $z$ direction in the half-space $z>-h$. The unknown factor $F(l)$ is determined by the boundary condition (2.18). Substituting expressions (3.5) and (3.6) in this boundary condition by performing the $z$ derivations under the

${ }^{5}$ A. Sommerfeld, Partial Differential Equations in Physics (Academic Press, Inc., New York, 1949), p. 242.

${ }^{6}$ Lord Rayleigh, Theory of Sound (Dover Publications, New York, 1945), Vol. II, p. 317. integral sign and putting $z=0$ we find

$$
-F(l)=\frac{1}{2} \sigma[2 \mu D+\mu F(l)]+\frac{1}{2} \sigma k^{2}\left[\frac{2 D}{\mu}+\frac{F(l)}{\mu}\right] .
$$

Solving for $F(l)$,

$$
F(l)=-\frac{\sigma l^{2}}{\mu+\frac{1}{2} \sigma l^{2}} D .
$$

Hence the velocity potential of the total reflected field $\varphi_{i}+\varphi$ including the effect of the roughness is,

$$
\varphi_{i}+\varphi=D \int_{0}^{\infty} \frac{1}{\mu} \cdot \frac{\mu-\frac{1}{2} \sigma l^{2}}{\mu+\frac{1}{2} \sigma l^{2}} J_{0}(l r) e^{-\mu(\tau+h)} l d l .
$$

We will now clarify the physical nature of this field by showing that it may be represented by distributed image sources.

\section{REPRESENTATION OF THE REFLECTED WAVE BY DISTRIBUTED IMAGE SOURCES}

We have by definition

$$
l^{2}=\mu^{2}+k^{2}
$$

hence the fraction in the integrand of (3.9) may be written

$$
\frac{\mu-\frac{1}{2} \sigma l^{2}}{\mu+\frac{1}{2} \sigma l^{2}}=-\frac{\sigma \mu^{2}-2 \mu+\sigma k^{2}}{\sigma \mu^{2}+2 \mu+\sigma k^{2}} .
$$

Considering $\mu$ as a variable this expression may be expanded in partial fractions in the form

$$
\frac{\mu-\frac{1}{2} \sigma l^{2}}{\mu+\frac{1}{2} \sigma l^{2}}=-1+\frac{A_{1}}{\mu+\mu_{1}}+\frac{A_{2}}{\mu+\mu_{2}},
$$

where $-\mu_{1}$ and $-\mu_{2}$ are the roots of the quadratic equation in $\mu$

$$
\sigma \mu^{2}+2 \mu+\sigma k^{2}=0
$$

we have

$$
\begin{aligned}
& \mu_{1}=\underset{\sigma}{\stackrel{1}{\sigma}}\left[1-\left(1-\sigma^{2} k^{2}\right)^{\frac{1}{2}}\right] \\
& \mu_{2}=\frac{1}{\sigma}\left[1+\left(1-\sigma^{2} k^{2}\right)^{\frac{1}{2}}\right] .
\end{aligned}
$$

Because of our initial assumption that the radius $a$ of the bosses is small relative to the wavelength, $\sigma k$ is small and values of $\mu_{1}$ and $\mu_{2}$ are real and positive. The coefficients in expressions (4.3) are

$$
\begin{aligned}
A_{1} & =-\frac{4}{\sigma} \cdot \frac{\mu_{1}}{\mu_{1}-\mu_{2}} \\
A_{2} & =\frac{4}{\sigma} \cdot \frac{\mu_{2}}{\mu_{2}-\mu_{1}} .
\end{aligned}
$$


Replacing the fraction (4.2) in the integrand of (3.9) by its expansion (4.3) in partial fraction the representation of the reflected field splits up into three terms

where

$$
\varphi_{i}+\varphi=\varphi_{1}+\varphi_{2}+\varphi_{3},
$$

$$
\begin{aligned}
& \varphi_{1}=-D \int_{0}^{\infty} \frac{1}{\mu} J_{0}(l r) e^{-\mu(z+h)} l d l \\
& \varphi_{2}=D A_{1} \int_{0}^{\infty} \frac{1}{\mu} \cdot \frac{1}{\mu+\mu_{1}} J_{0}(l r) e^{-\mu(z+h)} l d l \\
& \varphi_{3}=D A_{2} \int_{0}^{\infty} \frac{1}{\mu} \cdot \frac{1}{\mu+\mu_{2}} J_{0}(l r) e^{-\mu(z+h)} l d l .
\end{aligned}
$$

The first term $\varphi_{1}$ is simply the negative specular image

$$
\varphi_{1}=-D \frac{e^{-i k R_{i}}}{R_{i}}
$$

Consider now the second term $\varphi_{2}$ and let us write the Sommerfeld integral for a source located at a depth $h_{1}$ below the reflecting plane, we have

$$
\frac{e^{-i k R_{1}}}{R_{1}}=\int_{0}^{\infty} \frac{1}{\mu} J_{0}(l r) e^{-\mu\left(z+h_{1}\right)} l d l
$$

with

$$
R_{1}=\left[r^{2}+\left(z+h_{1}\right)^{2}\right] \text {. }
$$

Multiply both sides of the Eq. (4.10) by $e^{-\mu_{1} h_{1}}$

$$
e^{-\mu_{1} h_{1}} \frac{e^{-i k R_{1}}}{R_{1}}=\int_{0}^{\infty} \frac{1}{\mu} J_{0}(l r) e^{-\mu z} e^{-\left(\mu+\mu_{1}\right) h_{1}} l d l .
$$

The value of $\mu_{1}$ being positive we may integrate this equation with respect to $h_{1}$ between the limits $h_{1}=h$ to $h_{1}=\infty$ we write,

$$
\begin{aligned}
\int_{h}^{\infty} e^{-\mu_{1} h_{1}} \frac{e^{-i k R_{1}}}{R_{1}} d h_{1} \\
\quad=\int_{0}^{\infty} \frac{1}{\mu} J_{0}(l r) e^{-\mu z} l d l \int_{h}^{\infty} e^{-\left(\mu+\mu_{1}\right) h_{1}} d h_{1} \\
=e^{-\mu_{1} h} \int_{0}^{\infty} \frac{J_{0}(l r)}{\mu\left(\mu+\mu_{1}\right)} e^{-\mu(z+h)} l d l
\end{aligned}
$$

We recognize on the right-hand side the integral representing the value of $\varphi_{2}$. Hence

$$
\varphi_{2}=D A_{1} \int_{h}^{\infty} e^{-\mu_{1}\left(h_{1}-h\right)} \frac{e^{-i k R_{1}}}{R_{1}} d h_{1} .
$$

This represents the radiation of a continuous distribution of sources located on the $z$ axis below the specular image. The density of the distribution dies out exponentially with depth proportionally to the factor $\exp \left[-\mu_{1}\left(h_{1}-h\right)\right]$. The field $\varphi_{3}$ results similarly from an exponential distribution of sources with a different exponential factor $\exp \left[-\mu_{2}\left(h_{1}-h\right)\right]$.

$$
\varphi_{3}=D A_{2} \int_{h}^{\infty} e^{-\mu_{2}\left(h_{1}-h\right)} \frac{e^{-i k R_{1}}}{R_{1}} d h_{1} .
$$

\section{DISCUSSION OF THE RESULTS}

Let us consider the case of a vanishingly small roughness parameter $\sigma$. Expressions (4.5) and (4.6) become

$$
\begin{array}{ll}
\mu_{1}=\frac{1}{2} \sigma k^{2} & A_{1}=\sigma k^{2} \\
\mu_{2}=2 / \sigma & A_{2}=4 / \sigma .
\end{array}
$$

With these values $\varphi_{2}$ may be written

$$
\varphi_{2}=D \sigma k^{2} \int_{0}^{\infty} e^{-\downarrow j \sigma k^{2}\left(h_{1}-h\right)} \frac{e^{-i k R_{1}}}{R_{1}} d h_{1}
$$

while $\varphi_{3}$ is

$$
\varphi_{3}=\frac{4 D}{\sigma} \int_{0}^{\infty} \exp \left[-\frac{2}{\sigma}\left(h_{1}-h\right)\right]_{R_{1}}^{e^{-i k R_{1}}} d h_{1} .
$$

It is easily seen that if $\sigma$ tends to zero this latter expression becomes identical with a source equal to twice the specular image, i.e.,

$$
\varphi_{3}=2 D \frac{e^{-i k R_{i}}}{R_{i}}
$$

Hence the total reflected field becomes

$$
\varphi_{i}+\varphi=D \frac{e^{-i k R_{i}}}{R_{i}}+\varphi_{2},
$$

namely it tends to that of a specular image and a vanishingly small residue $\varphi_{2}$. It is interesting to note that, while the residual field $\varphi_{2}$ becomes vanishingly small, it is the result of sources which are distributed on the $z$ axis below the specular image with an exponential density which decreases very slowly with depth. This means that for a pulse signal the residual field corresponds to small intensity echoes with very large time delays after the first arrival.

Another limiting case of interest is represented by the intensity of the reflected field at large distances and in particular, at grazing incidence. In order to evaluate the field at large distance we put

$$
h_{2}=h_{1}-h
$$

and write

$$
R_{1}=R_{i}\left[1+2\left(h_{2} / R_{i}\right) \cos \theta+\left(h_{2}^{2} / R_{i}^{2}\right)\right]^{\frac{1}{2}} .
$$


This quantity is the distance of the observer $O$ to a point $P$ located at a distance $h_{2}$ below the specular image $I$, while $\theta$ is the angle of the line $O I$ with the vertical. Hence, $\theta$ is the angle of incidence for specular reflection. We may write

$$
\cos \theta=(z+h) / R_{i} .
$$

Consider now the following exponent in the integrand of (4.13)

$$
\mu_{1}\left(h_{1}-h\right)=\mu_{1} h_{2}=\mu_{1} R_{i}\left(h_{2} / R_{i}\right) .
$$

At large distance it is only in the range of small values of $h_{2} / R_{i}$ that the exponential factor contributes to the value of the integral. For small values of $h_{2} / R_{i}$ we may write the approximation

$$
R_{2}=R_{i}+h_{2} \cos \theta
$$

Hence expression (4.13) for $\varphi_{2}$ becomes

$$
\varphi_{2}=D A_{1} \frac{e^{-i k R_{i}}}{R_{i}} \int_{0}^{\infty} e^{-\left(\mu_{1}+i k \cos \theta\right) h_{2}} d h_{2}
$$

and by integrating,

$$
\varphi_{2}=\frac{D A_{1}}{\mu_{1}+i k \cos \theta} \cdot \frac{e^{-i k R_{i}}}{R_{i}} .
$$

Similarly,

$$
\varphi_{3}=\frac{D A_{2}}{\mu_{2}+i k \cos \theta} \cdot \frac{e^{-i k R_{i}}}{R_{i}} .
$$

The total reflected field at large distance is

$$
\begin{aligned}
\varphi_{i}+\varphi=\varphi_{1}+\varphi_{2}+\varphi_{3}=D\left[-1+\frac{A_{1}}{\mu_{1}+i k \cos \theta}\right. \\
\left.+\frac{A_{2}}{\mu_{2}+i k \cos \theta}\right] \frac{e^{-i k R_{i}}}{R_{i}} .
\end{aligned}
$$

Referring to relation (4.3) we see that the quantity between brackets is simply the fraction (4.2) in which $\mu$ has been replaced by $i k \cos \theta$. Hence,

$$
\varphi_{i}+\varphi=D \frac{2 \cos \theta+i \sigma k \sin ^{2} \theta}{2 \cos \theta-i \sigma k \sin ^{2} \theta} \frac{e^{-i k R_{i}}}{R_{i}} .
$$

The factor represents simply a change of phase angle of the source radiation without change of intensity. We put

$$
\frac{2 \cos \theta+i \sigma k \sin ^{2} \theta}{2 \cos \theta-i \sigma k \sin ^{2} \theta}=e^{2 i \psi} .
$$

The phase angle $2 \psi$ is given by

$$
\tan \psi=\frac{1}{2} \sigma k \frac{\sin ^{2} \theta}{\cos \theta} \text {. }
$$

The phase angle varies with the angle of incidence. For $\theta \rightarrow \pi / 2$, i.e., grazing incidence the phase angle $2 \psi$ tends to $180^{\circ}$. In this case the reflected and direct signals are of equal intensity and opposite phase hence they cancel each other near the reflecting surface. This results in a zone of silence at large distance in the vicinity of the reflecting surface. It is important to call attention to the fact that this phenomenon occurs no matter how small the radius $a$ of the bosses. However, as the roughness becomes vanishingly small the range of angles of incidences for which this effect is apparent is closer and closer to $90^{\circ}$.

In order to evaluate the orders of magnitudes involved, consider the complementary angles

$$
\begin{aligned}
\psi^{\prime} & =(\pi / 2)-\psi \\
\theta^{\prime} & =(\pi / 2)-\theta .
\end{aligned}
$$

The angle $\theta^{\prime}$ is the angle of the incident ray with the reflecting plane. Assuming $\theta^{\prime}$ to be small, Eq. (5.17) yields the approximation

$$
2 \psi^{\prime} \cong(4 / \sigma k) \theta^{\prime}
$$

For the field to cancel out the reflected and direct signals must be nearly out of phase, hence $2 \psi^{\prime}$ must be of the order of say $\pi / 4$. Hence,

$$
\theta^{\prime} \cong(\pi / 16) \sigma k \text {. }
$$

Let us assume a case where the hemispherical bosses of radius $a$ are located on the average at the vertices of a lattice of equilateral triangles with an average distance $3 a$ between their centers. Then two tri-angles of area $(9 / 2) \sqrt{3} a^{2}$ contain one boss. Therefore, the number of bosses per unit area is

$$
N=\frac{2}{9 \sqrt{3} a^{2}}
$$

and

$$
\sigma=2 \pi N a_{3}=\frac{4 \pi}{9 \sqrt{3}} a .
$$

If the wavelength of the source is $\lambda$ we find

$$
\sigma k=\frac{8 \pi^{2}}{9 \sqrt{3}} \underset{\lambda}{\stackrel{a}{2}} \stackrel{a}{\lambda}
$$

Hence,

$$
\theta^{\prime}=\frac{5 \pi}{16 \lambda} \underset{\lambda}{\stackrel{a}{\underline{\lambda}}}-
$$

If the wavelength is ten times the radius of the bosses $\theta^{\prime}=1 / 10$ and a region of silence occurs for rays which lie within about 5 degrees from the plane of reflection. For instance in air imagine the roughness to be the size of pebbles of radius $a=3 \mathrm{~cm}$, the wavelength 
corresponding to 5 degree silence zone is $30 \mathrm{~cm}$ and the frequency about $1 \mathrm{kc}$.

In the foregoing we have neglected the effect of the viscosity. It is clear that the viscosity creates a boundary layer near the surface of reflection where the velocity drops to zero as we approach the surface. The roughness effects which we have dealt with above will of course arise only if the bosses protrude outside the boundary layer, i.e., if the boundary layer thickness is small compared to the boss radius $a$. If the solid boundary were moving with the same tangential velocity $U$ as the fluid there would be no friction effect. The boundary layer therefore is close to that occurring in a fluid when a plane boundary oscillates harmonically with a tangential velocity of amplitude $U$ and angular frequency $\omega$. The velocity at a distance $z$ from the boundary is $\dagger$

with

$$
u=U e^{-\beta z} \cos (\omega t-\beta z)
$$

$$
\beta=(\omega / 2 \nu)^{\frac{1}{2}} .
$$

The kinematic viscosity of the fluid is denoted by $\nu=\eta / \rho(\eta=$ viscosity, $\rho=$ mass density). This velocity field dies out rapidly as we move away from the surface $z=0$. The thickness of the boundary layer may be taken to be the quarter-wavelength

$$
z_{1}=\pi / 2 \beta=\frac{1}{2}(\pi \nu / f)^{\frac{1}{2}},
$$

where $f=\omega / 2 \pi$ is the frequency.

The condition of validity of the present theory is

$$
z_{1} \ll a .
$$

Consider again the case $f=1 \mathrm{kc}$. For air at $15^{\circ} \mathrm{C}$ $\nu=0.125 \mathrm{~cm}^{2} / \mathrm{sec}$, hence

$$
z_{1}=10^{-2} \mathrm{~cm} \text {. }
$$

For water at $15^{\circ} \mathrm{C}, \nu=0.012 \mathrm{~cm}^{2} \mathrm{sec}^{-1}$, hence

$$
z_{1}=0.31 \times 10^{-2} \mathrm{~cm} \text {. }
$$

We see that except for extreme cases the effect of the roughness will not be modified appreciably by the viscosity.

\section{APPENDIX}

As mentioned in the text, when the distance between the bosses becomes of the order of their dimension there is a short-range interaction effect which may be taken into account by introducing a coefficient $\kappa$ in the definition of $\sigma$.

$$
\sigma=(2 \pi / \kappa) N a^{3}
$$

The value of this coefficient $\kappa$ may be evaluated very simply if the wavelength is assumed large $(k a \ll 1)$. In 6).

$\uparrow$ Solution derived by Stokes and given by Rayleigh (reference that case the flow in a region which is small relative to the wavelength is the same as for an incompressible fluid. The problem is then to find the dipole induced in a boss by a uniform flow $U_{x}$ parallel with the plane of reflection and taking into account the short range interaction of the surrounding bosses. The dipole moments induced in the bosses are parallel with $U_{x}$ and denoted by $M_{x}$. A boss located at the origin is surrounded on the average by six bosses. If the average distance between the center of the bosses is denoted by $b$ the six surrounding bosses are located at the vertices of an hexagon inscribed in a circle of radius $b$. The velocity induced at the origin by the six associated dipole moments is

$$
u_{x}^{(1)}=3 M_{x} / b^{3} .
$$

This value is the same whether we consider concentrated dipoles or whether we distribute them uniformly over the circle. The next nearest row of dipoles is on the circle of radius $2 b$. Since there are twice as many dipoles the velocity induced at the origin by this second circle is

$$
u_{x}^{(2)}=\frac{1}{(2)^{2}} \frac{3 M_{x}}{b^{3}} .
$$

In general the velocity induced by the dipoles at the distance $n b$ is

$$
u_{x}^{(n)}=\frac{1}{(n)^{2}} \frac{3 M_{x}}{b^{3}} .
$$

The total induced velocity resulting from short-range interaction is

$$
U_{x}^{(i)}=\frac{3 M_{x}}{b^{3}}\left(1+\frac{1}{4}+\cdots \frac{1}{n^{2}}\right)=\frac{n^{2}}{2} \frac{M_{x}}{b^{3}} . \ddagger
$$

The total velocity which induces a dipole at the origin is

$$
U_{x}^{\prime}=U_{x}+U_{x}^{(i)} .
$$

Hence

$$
U_{x}^{\prime}=U_{x}+\frac{\pi^{2}}{2} \frac{M_{x}}{b^{3}}
$$

On the other hand, the magnitude of the induced dipole in the hemispherical boss of radius $a$ is

$$
M_{x}=-\frac{1}{2} a^{3} U_{x}^{\prime}
$$

Substituting in (A.7) we derive

$$
\kappa U_{x}^{\prime}=U_{x}
$$

$\ddagger$ For an evaluation of the series see Jahnke and Emde, Table of Functions (B. G. Teubner, Berlin, 1933), p. 322. 
with

$$
\kappa=1+\frac{\pi^{2} a^{3}}{4 b^{3}}
$$

The value of $\kappa$ converges rapidly toward unity if $b$ is appreciably larger than $a$. Consider an extreme case $b=3 a$, then $\kappa=1.091$. The correction is $9 \%$ in this case. The resultant reduction in the value of (A.1) of $\sigma$ represents the amount of shielding due to the neighboring bosses. For $b=4 \mathrm{a}$ the correction would only be $3.8 \%$.

\title{
Oppositely Directed Plane Finite Waves
}

\author{
R. D. FAY . \\ Acoustics Laboratory, Massachusetts Institute of Technology, Cambridge, Massachusetts
}

(Received July 31, 1957)

\begin{abstract}
The analysis is carried out in terms of particle velocities expressed as Mach numbers. In general, the pertinent quantities are expressed in relation to the infinitesimal values by a power series of these Mach numbers in which two powers are retained. To this order of approximation, the particle velocity is the vector sum of the particle velocities of the isolated component waves and the speed of propagation in each direction has an increment proportional to the vector difference of these particle velocities. The general behavior of the sound field may be predicted from these two relations.
\end{abstract}

\section{INTRODUCTION}

$\mathrm{T}$ $\mathrm{HE}$ method of attack on plane progressive finite waves previously reported ${ }^{1}$ has been adapted to the analysis of a sound field that comprises plane finite waves progressing in opposite directions. The general scheme of analysis has been retained in that relations are obtained among the instantaneous values of the quantities of interest. In general, these quantities are expressed to a degree of approximation one order higher than that appropriate to infinitesimal waves.

It has been found advantageous to modify the original method by expressing the basic energy and power relations in terms of the absolute pressure. It is also found that many of the effects produced by interference do not depend on energy conservation; the sequence in the analysis has been modified to take advantage of this fact.

\section{BASIC RELATIONS}

The first step is to formulate conservation criteria for two pulses of uniform amplitude but of infinitesimal length for conditions of isolation and of superposition. The situation is indicated in Fig. 1. The speeds of propagation of the isolated pulses are designated by $S_{1}$ and $S_{2}$. In the region of interference, these speeds become $S_{12}$ and $S_{21}$. At time $t=0$, the leading fronts of these pulses have just established contact as indicated in the figure. The lengths, that is to say, the volume per unit area, are chosen to have such values that at time $t=d t$ the pulses occupy the same space. Thus, at time $t=0$ the lengths are $\left(S_{1}+S_{21}\right) d t$ and $\left(S_{2}+S_{12}\right) d t$, and at time $t=d t$ both lengths are $\left(S_{12}+S_{21}\right) d t$.

\footnotetext{
${ }^{1}$ R. D. Fay, J. Acoust. Soc. Am. 28, 914 (1956).
}

The criterion for conservation of momentum is, therefore,

$$
\left(S_{12}+S_{21}\right) \rho v=\left(S_{1}+S_{21}\right) \rho_{1} v_{1}-\left(S_{2}+S_{12}\right) \rho_{2} v_{2},
$$

where $\rho$ and $v$ represent density and particle velocity in the common volume. Subscripts 1 and 2 designate isolated pulses. Speeds and velocities are relative to the undisturbed medium. Velocities are taken to be positive in the direction of propagation; the velocity, $v$, is taken to be positive in the direction of $v_{1}$.

Two mass elements, Fig. 1, are now designated such that at time $t=d t$ the space occupied by the elements coincides with the space occupied by the momentum. The common boundary of these elements has moved a distance $v d t$ in time $d t$. The other boundaries, as indicated in the figure, have moved distances $v_{1} d t$ and $v_{2} d t$.

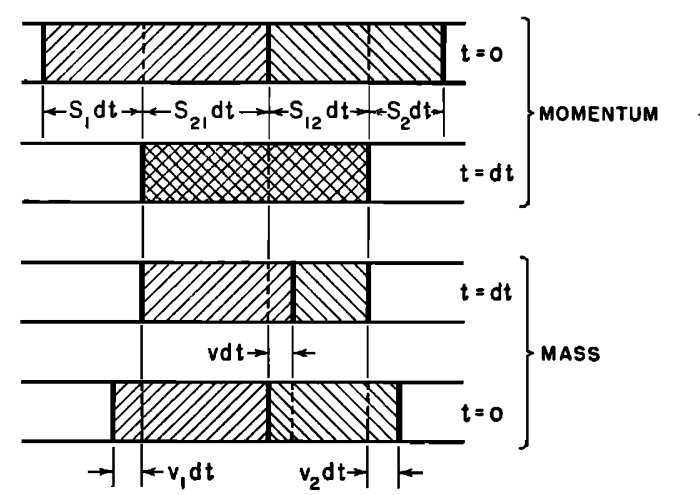

FIG. 1. The cross-hatched areas indicate volumes per unit area of wave front associated with the criteria for conservation of momentum and of mass. 